\title{
Applicability of Genetic Algorithms to Reconstruction of Projected Data from Ultrasonic Tomography
}

\author{
Shyam P. Kodali, Sunith Bandaru, K. Deb, Prabhat Munshi, N.N.Kishore \\ Department of Mechanical Engineering, Indian Institute of Technology Kanpur

$\begin{array}{ccccc}\text { 91-512-2597668 } & 91-512-2597668 & 91-512-2597205 & 91-512-2597243 & 91-512-2597049 \\ \text { kodalisp@iitk.ac.in } & \text { sunithb@iitk.ac.in } & \text { deb@iitk.ac.in } & \text { pmunshi@iitk.ac.in } & \text { nnk@iitk.ac.in }\end{array}$

\begin{abstract}
The use of a-priori information, where available, is an important step in solving an already computationally expensive tomographic imaging problem [1]. Here, an enhanced genetic algorithm based reconstruction technique is proposed that is capable of detecting the shape, size and location of multiple types of inclusions of known physical properties in a given test specimen. Preliminary results are found to be better than those reported with MART1. Simulations show that the algorithm is consistent for a wide range of grid sizes and geometries of inclusion(s). A logarithmic time complexity analysis gives a linear relationship between number of unknowns and reconstruction times, thus establishing the predictability of the algorithm.
\end{abstract}

\section{Categories and Subject Descriptors I.4.5 [Image Processing and Computer Vision]: Reconstruction.}

\section{General Terms}

Algorithms

\section{Keywords}

Block Crossover, Genetic Algorithms, Reconstruction, Time of Flight, Ultrasonic Tomography.

\section{INTRODUCTION}

Ultrasonic tomography provides an easy and cost effective way of detecting defects when the base material and inclusions are known to have approximately uniform characteristics. Acoustic wave attenuation and time of flight, as in the present case, are the two parameters which can be used for reconstruction. Popular reconstruction techniques include transform methods like Convolution Back Projection (CBP) and series expansion methods like Algebraic Reconstruction Technique (ART) and its variant multiplicative algebraic reconstruction technique (MART). The latter uses a discretized initial model. This transforms the problem from analytic to combinatorial. While transform methods require large sets of projection data, iterative methods suffer from ill-conditionality and multi-modality due to discretization and here lays the motivation for using genetic algorithms. They can handle continuous as well as discrete problems in almost the same way. Also since they work with a population, global convergence is most certainly ensured. Algebraic reconstruction techniques on the other hand, suffer from their inherent possibility of getting entrapped in a local optimum.

Copyright is held by the author/owner(s).

GECCO'08, July 12-16, 2008, Atlanta, Georgia, USA.

ACM 978-1-60558-130-9/08/07.

\section{DATA ACQUISITION}

The specimen is represented as a square matrix grid of integer values with each value corresponding to one type of material. In the following study, 0 corresponds to base material, and values 1 and 2 correspond to two different inclusion materials. The time of flight data is collected using the modified cross-hole geometry [2] configuration, where $\mathrm{N}$ transducers are equally spaced on each edge of the specimen. It is assumed that from each transducer one ray travels to each of the other transducers (except to those on the same edge as the source), thus giving us a total of $\mathrm{N}^{2}$ rays between any two edges. The simulated time of flight for a ray originating from $j$-th source and terminating at the $k$-th detector is estimated according to the relation,

$$
\text { simulated_tof }(j, k)=\sum_{m=1}^{M} \frac{l_{m}}{v_{m}}
$$

Where,

$M=$ Number of cells through which the ray passes,

$l_{m}=$ Length of the ray intercepted by $m$-th cell,

$v_{m}=$ Velocity of propagation of ultrasound through the $m$-th cell (depends on the material corresponding to that cell).

\section{RECONSTRUCTION ALGORITHM}

Through the use of a GA, we are looking for a particular distribution of the inclusion(s) which best agrees with the data obtained. To begin with, we generate a population of solutions with random distributions on a coarse grid. The idea is to proceed in steps towards the solution. The best possible solution with a relatively coarser initial grid serves as a seed for the next finer grid. Thus the core structure of the inclusions is identified in the initial steps while the later ones identify the boundaries.

To establish the superiority or inferiority of one population member over another, we define the following misfit function. During the GA process we try to emphasize members which have a low misfit value.

$$
\Phi(i)=\sum_{j=1}^{S} \sum_{k=1}^{D} \mid \text { actual_tof }(j, k)-\text { simulated_tof }(j, k) \mid
$$

Where,

$$
\begin{array}{ll}
\Phi(i) & =\text { Misfit of } i \text {-th population member } \\
\mathrm{S} & =\text { Number of sources } \\
\mathrm{D} & =\text { Number of detectors }
\end{array}
$$

While most of the binary GA process [3] remains essentially the same, the crossover and mutation are modified to suit specific needs. Elitism is introduced to conserve good members. 
The normal crossover operation of GA is modified to suit the two-dimensional nature of the population members. Instead of creating a single random number as the crossover site, four random numbers are created (two for each corner of sub-matrix). These sub-matrices are exchanged in the subsequent 'blockcrossover' operation.

Two different types of mutations have been incorporated into the reconstruction algorithm. The first is the more obvious 'bitwise-mutation', where individual cells are mutated. The second termed 'block-mutation' mutates the value in the selected cell and eight surrounding cells to the one that appears most number of times in these nine cells. During simulation runs it was found that bitwise mutations lose their efficacy for grid sizes greater than $30 \times 30$.

The resulting solution with the coarse grid is refined by splitting each cell into four and assigning them the same value as the parent cell. The cells corresponding to the core structure of the inclusion are now frozen. To identify such cells, the value in each cell is compared with those in the surrounding eight cells. If they all match, the cell under consideration is frozen. Values of cells that do not meet these criteria are unaltered. By randomly assigning values to the unfrozen cells, a new initial population is created. The base-population itself could be made a member of this new population.

\section{RESULTS AND DISCUSSIONS}

\subsection{Comparison with MART}

MART1 was used [2] to reconstruct a constant impulse field as shown in Figure 1 (a). It required eleven transducers per edge to obtain the image in Figure 1 (b), while a better image was produced as shown in Figure 1 (c) with only 3 transducers per edge with the proposed algorithm. This was the case with most reconstructions which shows the suitability of this new approach to limited data tomography.

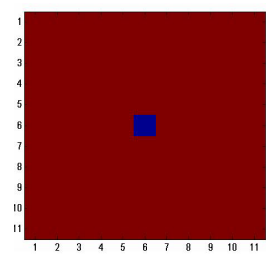

(a)

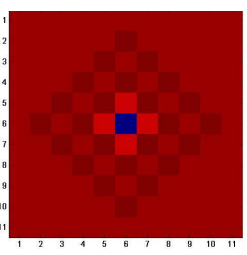

(b)

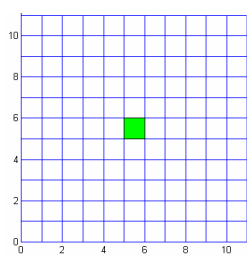

(c)
Figure 1. A comparison with MART

\subsection{Reconstructed Images}

The algorithm was tested for resolutions varying from $6 \times 6$ to $64 \times 64$ with combinations of different types of inclusions; in terms of material, shape, size, and location were performed. A representative result is illustrated in Figure 2.
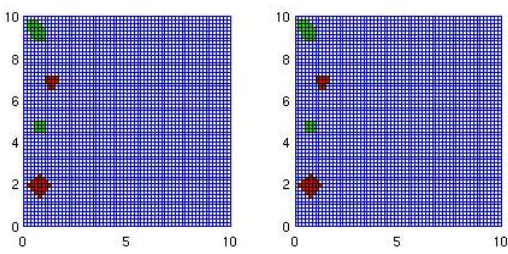

$\mathrm{RR}=60, \mathrm{SGS}=15, \mathrm{~T}=19, \mathrm{GEN}=1000$

Figure 2. A representative result
The left part is the actual specimen and the right is the reconstructed image. The resolution required (RR), starting grid size (SGS), number of transducers per edge (T), and number of generations per step (GEN) used are indicated. Figure 3 shows how increasing the number of generations per step leads to improvement in the reconstruction results.

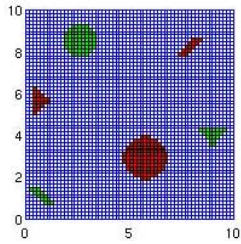

(a) Specimen

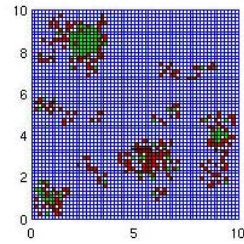

(b) GEN=1000

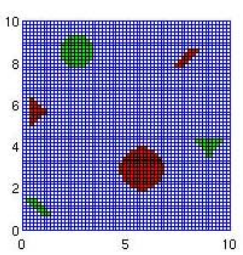

(c) $\mathrm{GEN}=\mathbf{2 5 0 0}$

$$
\mathrm{RR}=60, \mathrm{SGS}=15, \mathrm{~T}=19
$$

Figure 3. Effect of generations on convergence

\subsection{Reconstruction Times}

All reconstructions were performed on a SUN E250 machine with $400 \mathrm{MHz}$ dual processor and 1GB RAM using MATLAB 7 software. In Figure 4 the average reconstruction times for ten runs are plotted against the number of unknowns for reconstructing specimens with resolutions varying from $6 \times 6$ to $64 \times 64$. A single inclusion of square shape covering roughly $9-10$ percent of the specimen area and located at the centre of the specimen was reconstructed in each case. The relation between reconstruction times and number of unknowns is found to be nearly quadratic using which the reconstruction times for higher resolutions can be predicted.

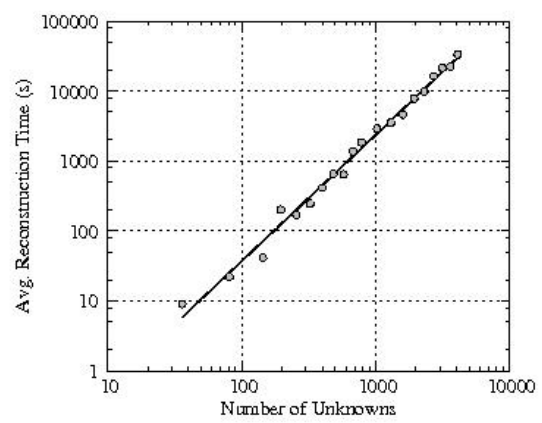

Figure 4. Plot showing the logarithmic linearity of the algorithm

\section{REFERENCES}

[1] P. P. Delsanto, A. Romano, M. Scalerandi and F. Moldoveanu, "Application of Genetic Algorithms to Ultrasonic Tomography", J. Acoustical Society of America, Vol. 104, No. 3, Pt. 1 (1998).

[2] Saurabh Khare, Mayuri Razdan, Prabhat Munshi, B. V. Soma Sekhar and K. Balasubramaniam, "Defect Detection in Carbon-Fiber Composites using Lamb-Wave Tomographic Methods", American Society for Nondestructive Testing, Research in Nondestructive Evaluation, Vol. 18, Issue 2, pp. 101-119 (2007).

[3] Kalyanmoy Deb, Optimization for Engineering Design: Algorithms and Examples, Prentice-Hall of India Pvt. Ltd. (2005). 